\title{
A RARE CASE OF ATYPICAL RENAL ARTERIES ARRANGEMENT WITH ECTOPIC KIDNEYS IN A GUINEA PIG
}

\author{
Maženský, D., Flešárová, S. \\ Department of Anatomy, Histology and Physiology \\ University of Veterinary Medicine and Pharmacy, Komenského 73, 04181 Košice \\ The Slovak Republic \\ david.mazensky@uvlf.sk
}

\section{ABSTRACT}

We recorded a very rare case of atypical renal arteries arrangement in a guinea pig using the corrosion technique in the study of the arterial system. The right renal artery originated from the ventral wall of the abdominal aorta at the level of the caudal aspect of the 5th lumbar vertebra. The left renal artery originated from the left common iliac artery approximately $12 \mathrm{~mm}$ caudally to the aortic bifurcation. The right kidney was located ventral to the aortic bifurcation and the left kidney inside the pelvic cavity between the common iliac arteries. According to the vascular pattern, we determined that the ectopic kidneys in this guinea pig were unusual. This is the first case describing bilateral ectopic kidneys in a guinea pig.

Key words: abdominal aorta; guinea pig; iliac artery; kidney; renal artery

\section{INTRODUCTION}

The general arrangement of renal arteries in the guinea pig is that they originate off of the lateral walls of the abdominal aorta caudal to the origin of the posterior mesenteric artery [10]. The literature describes several variations in the origin and arrangement of the guinea pig renal arteries [12]. In humans, they are found also as branches originating from various branches of abdominal aorta [3] or from the thoracic aorta [6]. The anomalous arrangement and origin of renal arteries is very often associated with unilateral or bilateral kidney ectopy.

Although a simple ectopic kidney is seldom responsible for symptoms, the association with malrotation of the renal pelvis with a calculus may increase the risk of hematuria, hydronephrosis, and stone formation with colicky pain [8]. Other signs and symptoms of ectopic kidneys include: incontinence, a palpable abdominal mass, urinary tract infection, renovascular hypertension secondary to an anomalous blood supply, and dystocia from a pelvic kidney [7]. An ectopic kidney is often associated with other abnormalities such as: agenesis of the opposite kidney, vascular 
malformation, and genital anomalies. Ectopic kidneys and an anomalous origin of the renal arteries were described in: the rat [5], dogs [4], a cat [2], a swine [11] and a bovine [9].

The purpose of this study is to describe a rare case of guinea pig ectopic kidneys using the corrosion technique of arterial system.

\section{MATERIALS AND METHODS}

In a routine study of the guinea pig arterial system, we found in one male an atypical position of the kidneys. The study of the arterial system in 26 adult English self guinea pigs (Cavia porcellus, L. 1758), aged 220 days was performed using the corrosion technique. We investigated guinea pigs of both sexes (female $n=13$; male $n=13$ ), in a weight range of $0.8-1 \mathrm{~kg}$, in an accredited experimental laboratory at the University of Veterinary Medicine and Pharmacy in Kosice. The animals were kept in cages under standard conditions (temperature $15-20^{\circ} \mathrm{C}$, relative humidity $45 \%, 12 \mathrm{~h}$ light period). A granular feed mixture (FANTASIA, Tatrapet, Liptovsky Mikulas, Slovak Republic) and drinking water were available to all animals ad libitum. The animals were sacrificed by intravenous injection of embutramide (T-61, $\left.0.3 \mathrm{ml} . \mathrm{kg}^{-1}\right)$. Immediately after euthanasia, the vascular network was perfused with a physiological solution. During the manual injection through the ascending aorta, the right atrium of the heart was opened in order to lower the pressure in the vessels in order to ensure an optimal injection distribution. Spofacryl (SpofaDental, Czech Republic) using a volume of $20 \mathrm{ml}$ was used as a casting medium. After polymerization of the medium, the maceration was carried out in $2-4 \% \mathrm{KOH}$ solution for a period of 2 days at $60-70^{\circ} \mathrm{C}$. This study was carried out under the authority of decision No. 2647/07-221/5.

\section{RESULTS}

The right renal artery originated from the ventral wall of the abdominal aorta at the level of the caudal aspect of the 5 th lumbar vertebra. The origin was located $4 \mathrm{~mm}$ cranial to the aortic bifurcation and $7 \mathrm{~mm}$ caudally to the origin of the caudal mesenteric artery. Furthermore, the right renal artery turned ventrally and caudally (Fig.1). During its course, in the place of contact of the right renal artery with the right kidney, it gave off two small interlobar arteries entering the kidney parenchyma. The main continuation entered the ventral directed renal hilus. The right kidney was located ventrally to the aortic bifurcation at the level from the caudal half of the 4th lumbar vertebra to the cranial half of the 6th lumbar vertebra (Fig. 2).

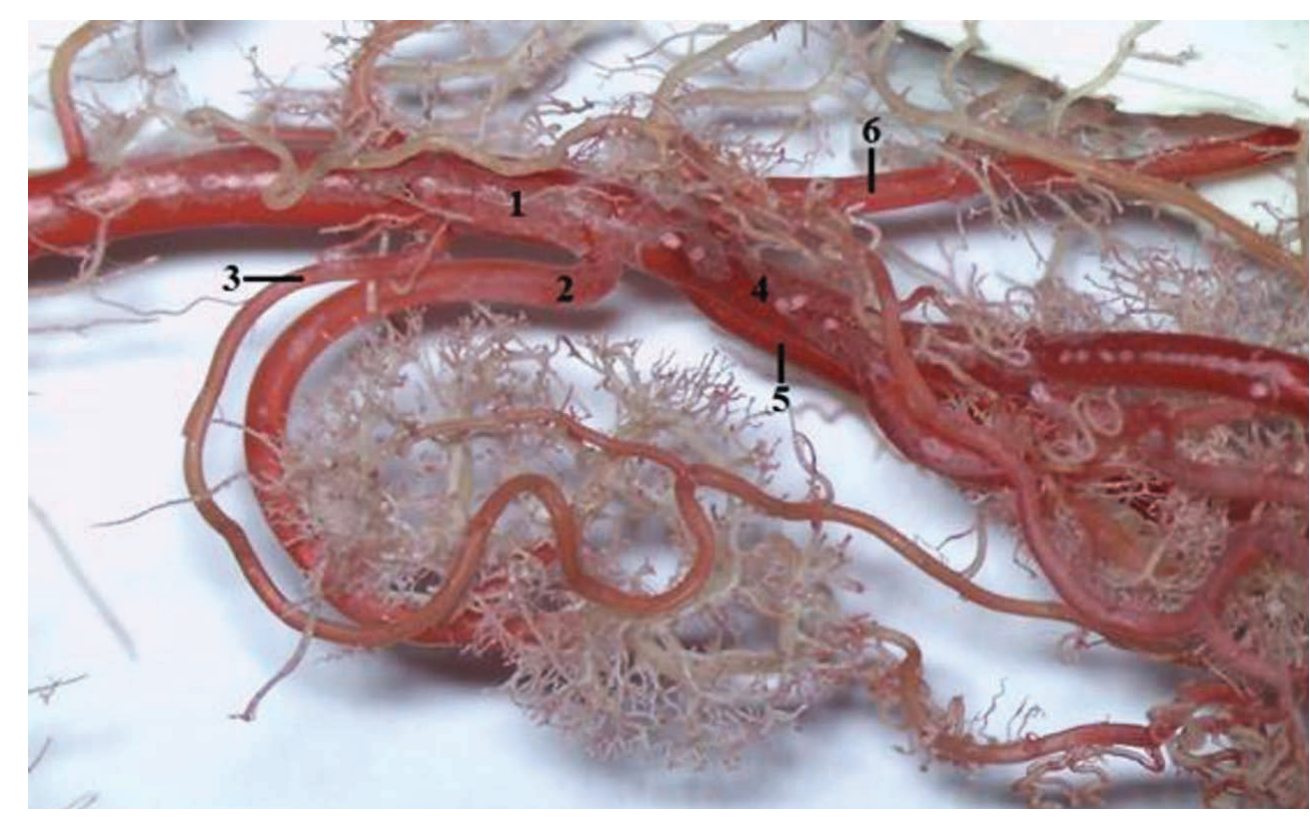

Fig. 1. Origin of the right renal artery. 1 - abdominal aorta; 2 - right renal artery; 3 - caudal mesenteric artery $4-$ left common iliac artery; $5-$ right common iliac artery; $6-$ median sacral artery. Left lateral view. Magn. $\times 3.2$ 


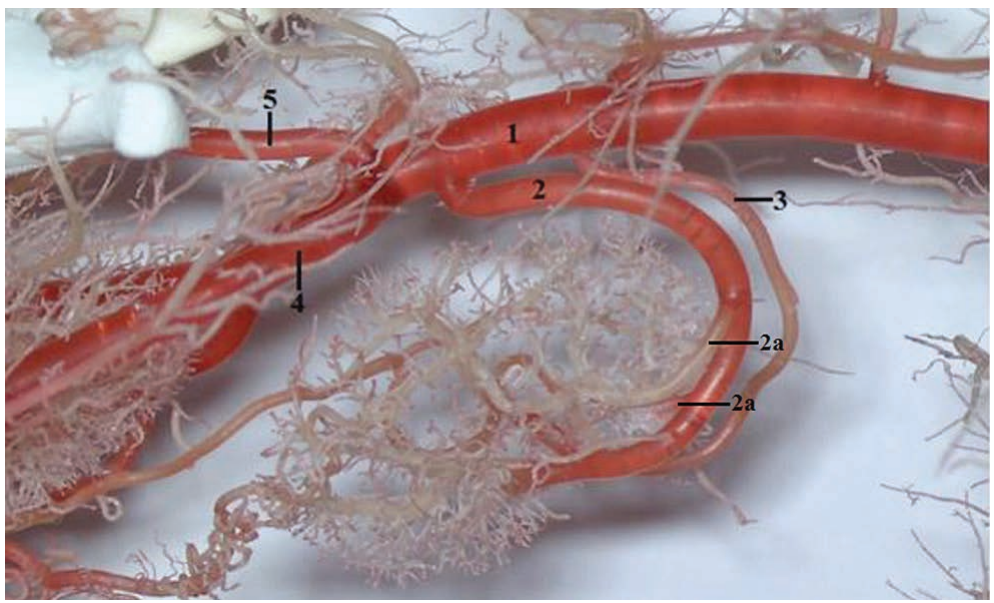

Fig. 2. Origin of the right renal artery. 1 - abdominal aorta; $2-$ right renal artery; $2 \mathrm{a}-$ interlobar arteries 3 - caudal mesenteric artery; $4-$ right common iliac artery; $5-$ median sacral artery. Right lateral view. Magn. $\times 3.2$

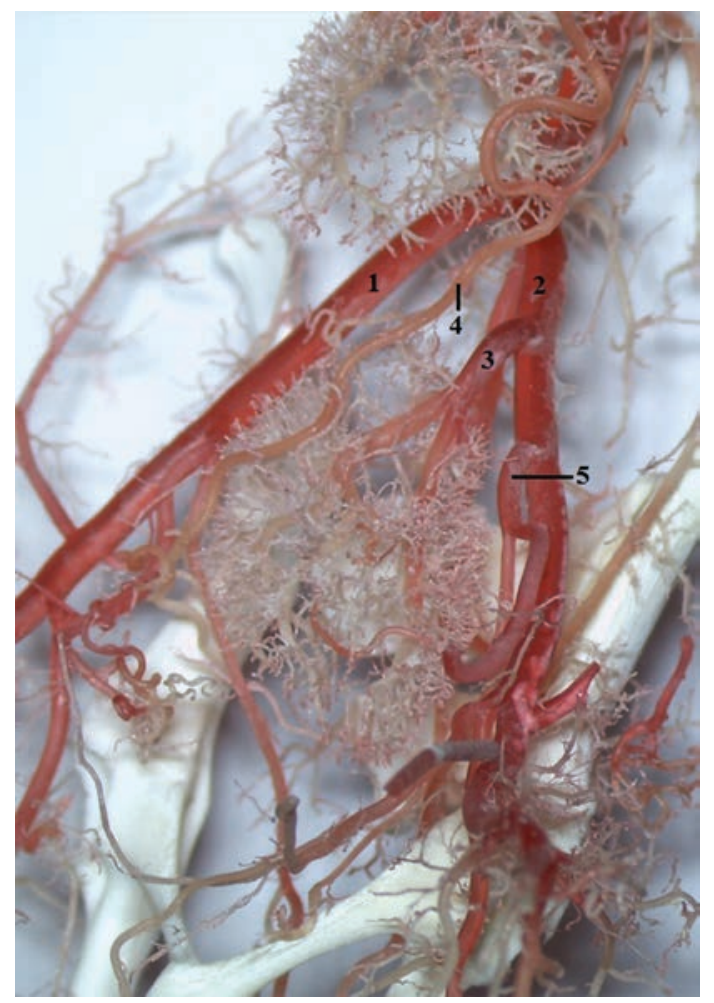

Fig. 3. Origin of the left renal artery. $1-$ right common iliac artery; $2-$ left common iliac artery; $3-$ left renal artery $4-$ caudal mesenteric artery; $5-$ left internal iliac artery. Ventral view. Magn. $\times 3.2$

The left renal artery originated from the left common iliac artery approximately $12 \mathrm{~mm}$ caudally to the aortic bifurcation and $8 \mathrm{~mm}$ cranially to the origin of the internal iliac artery. After running in a ventromedial direction, the left renal artery entered the left kidney through the craniolaterally positioned renal hilus. The position of the left kid- ney was inside the pelvic cavity between bilateral common iliac arteries (Fig. 3).

The cranial abdominal, adrenal and phrenic arteries were independent branches arising from the cranial part of the abdominal aorta. 


\section{DISCUSSION}

Po pesko et al. [10] described the origin of renal arteries in the guinea pig from the abdominal aorta by means of the cranial and caudal right renal artery, and cranial and caudal left renal arteries. The origin of the cranial renal arteries was found at the level of the 1st lumbar vertebra, and of the caudal renal arteries at the level between the 1st and 2nd lumbar vertebra. The cranial abdominal arteries originated independently from the abdominal aorta cranial to the cranial renal artery.

In about $20 \%$ of cases, both renal arteries were single, the right renal artery was double in over $50 \%$ of the cases, and the left renal artery was double in over $60 \%$ of the cases. Single or double renal arteries were divided into two branches before entering the kidney. The largest branch arising from the renal artery was the cranial abdominal artery. It branched from the cranial renal artery in cases of double renal arteries or just proximally to the division into branches in cases of single renal arteries [12].

An ectopic kidney usually does not show specific clinical signs. Unilateral ectopic kidneys are more common in several species of domestic mammals. In a cat, was described an ectopic right kidney while the left kidney was found in the correct place [2]. Ectopic kidneys were also described in dogs. In three dogs, was found in one case, bilateral ectopic kidneys and in two cases only a left ectopic kidney [4].

An ectopic kidney can cause poor outflow and is more susceptible to calculi, hydronephrosis and infection. Hydronephrosis is the most common finding in humans with an ectopic kidney [2]. There is also an increased incidence of other extrarenal malformations in humans, including skeletal anomalies (particularly vertebral), imperforate anus, cryptorchidism, other genitourinary and cardiovascular anomalies, and chromosomal defects [1].

\section{CONCLUSIONS}

The diagnosis of an ectopic kidney could be included in the differential diagnoses of abdominal or retroperitoneal masses. This is of particular importance, since ectopic kidneys are more susceptible to diseases than normal ones.
Although a clinically silent ectopic kidney is identified, careful monitoring of the patient may be needed in order to detect possible urologic problems.

\section{REFERENCES}

1. Allworth, M. S., Hoffmann, K. L., 1999: Crossed renal ectopy with fusion in a cat. Vet. Radiol. Ultrasound, 40, 357-360.

2. Brückner, M., Klumpp, S., Kramer, M., Thiel, C., 2010: Simple renal ectopy in a cat. Tierarztl. Prax. Kleintiere, 38, 163166.

3. Davidson, K. C., 1973: Unusual renal arteries in a potential kidney donor. Case report. Mo. Med., 70, 91.

4. Choi, J., Lee, H., Lee, Y., Choi, H., 2012: Simple ectopic kidney in three dogs. J. Vet. Med. Sci., 74, 1373-1375.

5. Gupta, B.N., 1975: Ectopic kidney in a rat. Lab. Anim. Sci., 25, 361

6. Ishida, M., Namiki, Y., Watanabe, M., 2016: Thoracic renal artery: a rare case of the renal artery originating from the thoracic aorta and literature review. Anat. Sci. Int., 91, 211-214.

7. Kang, I. J., Lee, S. H., Lee, S. M., Lim, C. H., et al., 2005: A case of congenital single ectopic kidney in pelvis of patients with proteinuria. Korean J. Nephrol., 24, 137-40.

8. Kumar, S., Bolla, S. R., Vollala, V. R., 2011: Unilateral ectopic kidney in the pelvis - a case report. Chang. Gung. Med. J., $34,10-12$.

9. Mueller, P. O., Hay, W. P., Allen, D., Collatos, C., Watson, E., 1999: Removal of an ectopic left kidney through a ventral midline celiotomy in a calf. J. Am. Vet. Med. Assoc., 15, 532-534.

10. Popesko, P., Rajtova, V., Horak, J., 1990: Anatomic Atlas of Small Laboratory Animals I (In Slovak). 1st edn., Priroda, Bratislava, $202 \mathrm{pp}$.

11. Sedan, J., 1956: Anophthalmia in swine with ectopic kidneys. Ann. Ocul. (Paris), 189, 392-401.

12. Shively, M. J., Stump, J.E., 1975: The systemic arterial pattern of the guinea pig: the abdomen. Anat. Rec., 182, 355-366.

Received August 24, 2016

Accepted September 9, 2016 